\title{
Could Serological Markers of the Dengue Viral Disease Predict the Outcomes in Pregnant South Asian Population?
}

\author{
${ }^{1}$ Dr. Harshita Guruprasad, Junior Resident, \\ ${ }^{2}$ Dr. Lakshmi Rachakonda, HOD Dept of OBGY \\ ${ }^{3}$ Dr. Shubhangi Mande, HOU, Dept of OBGY \\ MGM Medical College and Hospital , Aurangabad, Maharashtra, India.
}

Abstract

\section{$>$ Background:}

$A$ recent rise in the incidence of a vector borne viral disease, Dengue Disease, in South Asian countries has also shown an impact on the pregnant population in these regions. There are four known Dengue virus serotypes, the predominant ones being DENV- 2 and DENV-3, when present during pregnancy could be associated with complications like preterm birth, maternal mortality and morbidity and adverse neonatal outcomes.[1]. We aimed to study the outcomes in the pregnant patients that presented to our institution with the Dengue disease.

\section{$>$ Methods:}

A 6-month (1/9/2019 - 29/2/2020) study was undertaken during the months of high prevalence of the Dengue disease in our region. Clinical, laboratory, maternal and foetal outcomes were studied among serologically positive dengue mothers treated at MGM Hospital, Aurangabad.

\section{$>$ Results:}

A total of 25 patients were found to have Dengue disease during the study period. NS1Ag and IGM serological analysis results were used to achieve diagnosis and early management. 7 patients had symptomatic thrombocytopenia and needed platelet transfusions. Majority of our patients had favourable outcomes. 3 mothers died succumbing to the multiorgan failure.

\section{Conclusion:}

Favourable maternal and foetal outcomes could be achieved with early diagnosis of suspected Dengue disease with both NS1Ag and IGM serological analysis.

Keywords:- Dengue in Pregnancy, Outcomes of Dengue Fever, Serological Markers in Dengue.

\section{INTRODUCTION}

Dengue fever is a viral disease caused by any of the four closely related serotypes of genus Flavivirus, family Flaviviridae - a RNA virus. The four serotypes designated as DENV -1, DENV -2, DENV -3, DENV -4.[1] It is one of the most important vector borne diseases affecting countries like India, with the vector being the Aedes aegyptis mosquito. $40 \%$ of the world's population live in dengue prone zone, indicating the high risk and burden of carrying the disease. WHO estimates atleast 100 million infections occur every year including 5,00,000 dengue hemorrhagic fever cases and nearly 22,000 deaths.[2] The clinical severity of the disease has a wide spectrum and according to WHO Dengue classification there are four grades ranging from uncomplicated dengue fever (DF) to devastating Dengue Shock Syndrome (DSS). Infection by one serotype produces lifelong immunity to that specific serotype but only a few months of immunity to the others.[3]

Dengue infection in pregnancy carries the risk of haemorrhage for both the mother and the newborn. In addition, there is a risk of premature birth and fetal death and vertical transmission, which is well established, causing neonatal thrombocytopenia that necessitates platelet transfusions. [4-6]

Symptomatic dengue infection is a systemic and dynamic disease. The disease incubation period is around 2 weeks under favourable conditions. The clinical course of dengue infection passes through three phases -febrile phase, critical phase and recovery phase.[2] At parturition, severe bleeding may complicate delivery and/or surgical procedures performed on pregnant patients during critical phase of dengue i.e. marked thrombocytopenia . Literature search reveals that dengue is a significant factor for adverse pregnancy outcomes like preterm birth, Low birth weight, increased operative interference.[7] Diagnosis of dengue infection affects management and decisions of mode of delivery due to the hazardous risk of haemorrhage secondary to thrombocytopenia. Elevated liver enzymes, hemolysis, low platelet counts may be misleading and one might confuse it with HELLP syndrome. [9] Obstetrician being the frontline physician for a pregnant woman , should be able to identify, notify and manage cases of dengue fever in pregnancy. 
ISSN No:-2456-2165

There is insufficient data regarding the teratogenic power of the virus. As such dengue fever in pregnancy does not warrant termination of pregnancy.[10]

\section{Aim and Objectives}

- To analyse the clinical presentation of dengue fever in pregnancy.

- To correlate the course of the disease with serological markers.

- To study various maternal and neonatal outcomes .

\section{MATERIALS AND METHODS}

Study design - Prospective Observational study

Study period - September 2019 - February 2020. ( 6 months)

> Study place - MGM Medical College \& Hospital, Aurangabad, Maharashtra.

$>$ Sample size -25

$>$ Inclusion criteria - Pregnant females diagnosed and serologically confirmed to have dengue fever.

$>$ Patients were included irrespective of the period of gestation.

$>$ Exclusion criteria - other causes of fever among pregnant females.

$>$ Data was collected from the patient case records and hospital records of the confirmed cases.

A pre designed proforma was filled.

Data was analysed and interpreted.

\begin{tabular}{|l|l|}
\hline \multicolumn{1}{|c|}{ PARAMETER } & NUMBER OF PATIENTS ( N = 25) \\
\hline PARITY STATUS & \\
\hline 1. PRIMIGRAVIDA & $18(72 \%)$ \\
\hline 2. MULTIGRAVIDA & $07(28 \%)$ \\
\hline AGE OF THE MOTHER & \\
\hline 1. $<19$ YEARS & $03(12 \%)$ \\
\hline 2. $19-30$ YEARS & $21(84 \%)$ \\
\hline 3. $30-35$ YEARS & $01(4 \%)$ \\
\hline GESTATIONAL AGE AT DIAGNOSIS & \\
\hline 1. $<13$ WEEKS & $03(12 \%)$ \\
\hline 2. $13-28$ WEEKS & $14(56 \%)$ \\
\hline $3 .>28$ WEEKS & $08(32 \%)$ \\
\hline
\end{tabular}

Table 1:- Demographic Profile of Mother (Patient)

$72 \%$ of the patients were primigravida, since large number of patients booked at our hospital are primigravida. $84 \%$ of the patients were between 19-30 years of age, as it is mainly the reproductive age group. $56 \%$ of patients were in their second trimester followed by $32 \%$ in their third trimester. Almost all of these patients, diagnosed in second trimester could continue their pregnancy.

\begin{tabular}{|c|c|}
\hline CLINICAL SIGNS AND SYMPTOMS & NUMBER OF PATIENTS $(n=25)$ \\
\hline \multicolumn{2}{|l|}{ SYMPTOMS } \\
\hline 1. FEVER WITH CHILLS & $23(92 \%)$ \\
\hline 2. FEVER WITHOUT CHILLS & $01(4 \%)$ \\
\hline 3. MYALGIA & $20(80 \%)$ \\
\hline 4. HEADACHE & $11(44 \%)$ \\
\hline 5. PERSISTENT VOMITING & $04(16 \%)$ \\
\hline 6. ABDOMINAL PAIN & $03(12 \%)$ \\
\hline 7. BLEEDING DIATHESIS & $02(8 \%)$ \\
\hline 8. PETECHIAE & $00(0 \%)$ \\
\hline 9. SKIN RASH & $02(8 \%)$ \\
\hline \multicolumn{2}{|l|}{ SIGNS } \\
\hline 1. FEVER ( TEMP $\left.>100^{\circ} \mathrm{F}\right)$ & $14(56 \%)$ \\
\hline 2. TACHYCARDIA & $19(76 \%)$ \\
\hline 3. HYPOTENSION & $03(12 \%)$ \\
\hline 4. PRESENCE OF RASH & $02(8 \%)$ \\
\hline
\end{tabular}

Table 2:- Clinical Profile of Patients with Dengue Fever 
The following table shows the clinical presentation of patients at the time of admission. In our study, 92\% of mothers presented with fever with chills which was the most common presentation of dengue fever in pregnancy. Myalgia and headache were the next common symptoms with incidence of $80 \%$ and $44 \%$ respectively. Inspite of 52\% of patients having thrombocytopenia, only two patients presented with bleeding tendency in the form of malena and none of the patients presented with petechiae.

Clinically, at the time of admission, nearly $50 \%$ of the patients were documented to have fever with temperature above $100^{\circ}$ F. Tachycardia was noted in $76 \%$ of the patients with a resting heart rate above 100 beats per minute, in those with or even without fever.

\section{BIOLOGICAL TEST}

\section{SEROLOGICAL MARKERS}

1. DENGUE NS1 + IgM -

2. DENGUE NS1 - IgM +

3. DENGUE NS1 + IgM +

\section{SEVERITY OF THROMBOCYTOPENIA ( PLATELETS/CUMM)}

1. $<20,000$

2. $20,000-50,000$

3. $50,000-1,00,000$

4. $1,00,000-1,50,000$

5. $>1,50,000$

\section{OTHER LABORATORY TEST}

1. FALLING PLATELET COUNT

2. RISING HEMATOCRIT

3. LEUCOPENIA

4. DERANGED LFT

\section{NUMBER OF PATIENTS ( $n=25)$}

\section{$14(56 \%)$}

$6(24 \%)$

$5(20 \%)$
7 (28\%)

$3(12 \%)$

$3(12 \%)$

$3(12 \%)$

$9(36 \%)$
$20(80 \%)$

$15(60 \%)$

$08(32 \%)$

$07(28 \%)$

Table 3:- Laboratory Profile of Patients

$76 \%$ of patients tested positive for Dengue NS1Ag. Out of these $76 \%$ patients, nearly $56 \%$ patients tested positive only for $\mathrm{NS} 1 \mathrm{Ag}$ and the rest of them tested positive for Dengue IgM antibody as well. Only IgM positivity with negative results for NS1 Ag was noted among $24 \%$ patients. Both tested positive in nearly $20 \%$ patients, who had more severe platelet falling trend but clinically remained unaffected.

Nearly $80 \%$ of our patients had falling trend of platelets, though they did not necessarily qualify for the definition of thrombocytopenia $(<1,00,000 /$ cumm). Although $28 \%$ of patients had deranged LFTs, six patients had ICU admission and one patient was clinically stable, monitored in the ward. 


\begin{tabular}{|c|c|}
\hline OUTCOME & NUMBER OF PATIENTS ( $n=25$ ) \\
\hline \multicolumn{2}{|l|}{ DELIVERY STATUS } \\
\hline 1. VAGINALLY DELIVERED & 04 (16\%) \\
\hline 2. INTRAUTERINE DEATH & $02(8 \%)$ \\
\hline 3. PREGNANCY CONTINUED & $20(80 \%)$ \\
\hline \multicolumn{2}{|l|}{ MATERNAL OUTCOME } \\
\hline 1. RECOVERED & $22(88 \%)$ \\
\hline 2. MULTIORGAN FAILURE & $05(25 \%)$ \\
\hline 3. DEATH DUE TO MULTIORGAN FAILURE & $03(12 \%)$ \\
\hline \multicolumn{2}{|l|}{ NEONATAL OUTCOME } \\
\hline 1. NUMBER OF DELIVERIES & $04(16 \%)$ \\
\hline 2. NUMBER OF LIVEBIRTHS & $03(12 \%)$ \\
\hline 3. NUMBER OF IUD & $02(8 \%)$ \\
\hline
\end{tabular}

Table 4:- Outcome of Pregnancy and Neonates

Four patients (16\%) who delivered vaginally, did not land up in Post Partum Hemorrhage due to strong anticipation, aggressive third stage management and prophylactic transfusion in two patients (8\%). Strict fourth stage monitoring was done. But eventually two patients succumbed to multiorgan failure. One patient had IntraUterine Foetal Demise, succumbed before delivery.

\section{Flowchart}

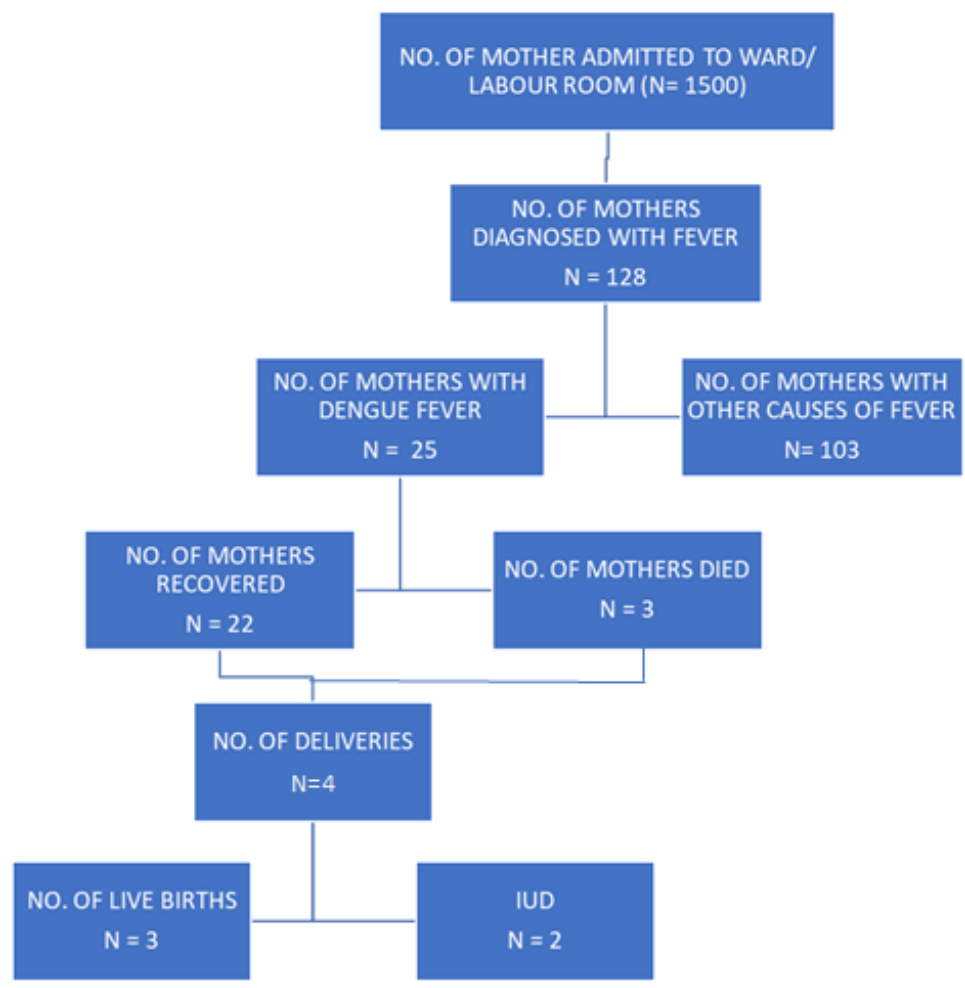

Fig 1

\section{DISCUSSION}

The outbreak of dengue is common during the monsoon and post monsoon season in the tropical belt. Dengue is endemic to the tropical areas and rare in temperate regions. Recently, there is rising epidemic potential of dengue. A high index of suspicion is needed in any pregnant female from these areas with fever during the epidemics. As reported previously, most cases of dengue fever during pregnancy had favourable outcomes. Therefore, most of the mothers could continue their pregnancy similar to the study carried out by Sampath Kariyawasam et.al.,.[7] Although our patients did not fulfil all the clinical and investigative criteria of dengue fever as defined by WHO, they had fever with thrombocytopenia. $56 \%$ of women in our study were in second trimester, 
contradictory to previous studies like Gehlot H.et.al., in whom incidence was higher in third trimester Almost all of these patients diagnosed in second trimester could continue pregnancy as they were remote from term and complications are expected when closer to term or in labour. As expected, four patients closer to their expected date of delivery had more severe clinical symptoms and landed up in multiorgan failure.[1] Most common symptoms included fever with chills( $92 \%)$,followed by myalgia(80\%) and arthralgia similar to other studies.[8] Though $76 \%$ of patients tested positive for NS1Ag which is highest among our patients, not all of them tested positive but $24 \%$ of patients tested positive only for $\operatorname{IgM}$ Antibodies, suggesting that the patients had recent infection with ending of viremic phase. In our study 25 pregnant females were included, after they serologically tested positive to the disease. So routine usage of NS1Ag testing as screening might not pick all the dengue positive patients, might give false negative results and hence, further coupling of testing by ELISA might be needed in patients with high index of suspicion. Out of the 25 patients , 7 patients had their platelets count dropped below 20,000 and needed platelet transfusions as they were symptomatic. All of the patients had a falling trend of the platelets, but values remained above critical level and 18 of them were asymptomatic. Fever with chills was noted to be the most common clinical presentation at the time of admission and maternal tachycardia with baseline heart rate above 100 beats per minute being the most common clinical sign noted in $76 \%$ patients. $6(24 \%)$ patients needed ICU admission in view of dengue shock syndrome and needed critical care.All those (100\%) who tested positive to both NS1Ag and IgM developed significant thrombocytopenia and multiorgan failure, including Acute Respiratory Distress Syndrome(ARDS) similar to that described by Lum et.al. Hence, these serological markers might aid in predicting the clinical course of the disease amongst pregnant females, and aid in better anticipation of complications and preparednessof the attending medical team. Four patients who delivered vaginally did not land up in $\mathrm{PPH}$ due to strong anticipation and aggressive management of third stage of labour. Elective LSCS was avoided in these patients to avoid any invasive procedures during the critical phase of dengue. One of the mothers had an Intrauterine death of the foetus and succumbed before delivery. There were a total of three live births and all three babies required NICU admission for observation and platelet monitoring. Stringent postpartum care , Hydration and supportive care reduce the maternal mortality and morbidity as shown by other studies

\section{CONCLUSION}

This study highlights the clinical pattern and the natural course of dengue in pregnancy. The clinical presentation of dengue in pregnancy is almost similar to dengue in non - pregnant adults but the clinical course might be dreadful with complications like Post Partum Haemorrhage, Disseminated Intravascular Coagulation, shock. Most of our patients could continue their pregnancy with better maternal outcomes due to proper monitoring during the antenatal period as well as intranatal period and adequate care offered at our centre. Easy and quick availability of blood and blood products, anticipation and adequate preparedness helped in achieving better outcomes. Dengue affection with serious consequences was seen in last trimester, as it complicated labour and was associated with both NS1Ag as well as $\operatorname{IgM}$ positivity. Hence serological markers could be used to predict severity of clinical course of dengue fever in pregnancy. Conservative management to be done unless there are complications or indications and till the critical phase of dengue is passed. Availability of Intensive care and efficient team work , aid in reducing the maternal morbidity and mortality and improved neonatal outcomes. Health care providers should consider dengue in differential diagnosis of pregnant women with fever during epidemics in endemic areas.

\section{REFERENCES}

[1]. Hanslata Gehlot, Om Prakash Yadav et,al. A study of dengue fever in pregnancy and its maternal and fetal prognosis.International Journal of Reproduction, Contraception, Obstetrics \& Gynaecology 2017 Aug;6(8):3414-3417

[2]. WHO . Dengue : guidelines for diagnosis , treatment, prevention and control. New edition 2009

[3]. Gibbons RV and Vaughn DW(2002) Dengue : an escalating problem. BMJ 324: 1563 - 1566

[4]. Bharaj P, Chahar HS, Pandey A, Diddi K, Dar L, Guleria R, Kabra SK, Broor S (2008)Concurrent infections by all four dengue virus serotypes during an outbreak of dengue in 2006 in Delhi. Virol J $9: 1$.

[5]. Carroll ID, Toovey S, Van Gompel A (2003)Dengue fever and pregnancy - a review and comment. Travel Med Infect Dis 5: $183-188$.

[6]. Chotigeat U, Kalayanrooj S, Nisalak A (2003) Vertical transmission of dengue infection in Thai infants : Two case reports. J Med Assoc Thai 86: 628 $-632$.

[7]. Sampath Kariyawasam, Hemantha Senanayake Dengue infections during pregnancy: case series from a tertiary care hospital in Sri Lanka, J Infect Dev Ctries 2010;4(11):767-775.

[8]. malavige GN, Velhanthiri VG, Patterns of disease among adults hospitalized with dengue infections.QJM 99: 299-305

[9]. Chitra TV, Panicker S. Maternal and fetal outcome of dengue fever in pregnancy . J Vector Borne Dis.2011;48(4):210

[10]. N Bhattacharya , H Mukherjee , R Naskar, S Talukdar. Serological diagnosis of dengue in laboratory practice in Kolkata. Indian $\mathbf{J}$ Med Microbiol.2014;32(3):277-80 